\begin{tabular}{l|c|c}
\hline ISSN: 0001-5113 & ACTA ADRIAT., & ORIGINAL SCIENTIFIC PAPER \\
AADRAY & $60(1): 47-52,2019$ & \\
\hline
\end{tabular}

\title{
A new record of the genus Trygaeus Dohrn, 1881 and species Trygaeus communis Dohrn, 1881 (Arthropoda, Pycnogonida) from Turkey (Eastern Mediteranean)
}

\author{
Cengiz KOCAK \\ Department of Hydrobiology, Fisheries Faculty, Ege University, TR 35100, \\ Bornova-Izmir, Turkey
}

Corresponding author, e-mail: kocakcengiz@gmail.com

Sampling studies in the Turkish Aegean Sea revealed the occurrence of the sea spider species,
Trygaeus communis Dohrn, 1881. The genus Trygaeus and species Trygaeus communis Dohrn,
1881 are recorded for the first time from Turkey. The genus and species are also recorded for the
second time from the Eastern Mediterranean Sea. Distribution map of the species in the Eastern
Mediterranean Sea is provided, together with photographs and line drawing of the species. This
record further extends the known distribution of the species to Turkey in the Eastern Mediterranean.

Key words: Pycnogonida, Trygaeus communis, Turkey, Eastern Mediterranean, Mediterranean Sea

\section{INTRODUCTION}

Trygaeus communis Dohrn, 1881 is endemic to the Mediterranean Sea and the only species in the genus Trygaeus. T. communis has occasionally been found mainly in western and central regions of the Mediterranean Sea (ARNAUD, 1987; CHIMENZ \& LATTANZI, 2003). In the Eastern Mediterranean Sea, it was reported only from Crete Island (Greece) by ARNAUD (1987). To date, there is no record of the genus Trygaeus from Turkey. An examination of sea spiders collected in Mordoğan (İzmir Bay, Turkish Aegean Sea) revealed the presence of a species belonging to the genus Trygaeus Dohrn, 1881, which is identified in this paper as T. communis.

The presence of the genus and species are reported for the first time from Turkey and the second time from the Eastern Mediterranean Sea.

\section{MATERIAL AND METHODS}

By snorkelling, one specimen of the species was collected from Cystoseira mediterranea Sauvageau, 1912 facies in the upper infralittoral zone (0.5 m depth) of Mordoğan, İzmir Bay (Aegean Sea, Turkey) (Fig. 1). The specimen was fixed in 5\% formaldehyde,later rinsed with fresh water and then preserved in $70 \%$ ethanol. The specimen was examined under a stereomicroscope. Drawing was made with the aid of a drawing tube mounted on an Olympus CX31 compound microscope. The following papers were used for the species identification: STOCK (1968), LEHMANN et al. (2014) and MUNILLA \& 


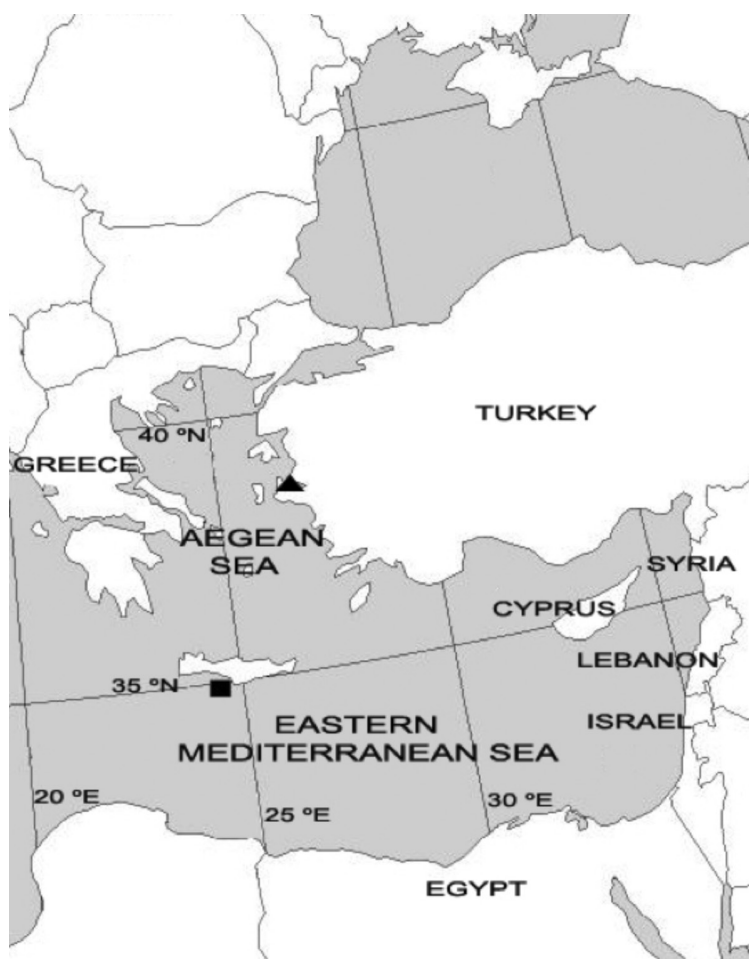

Fig. 1. Earlier established occurrence of Trygaeus communis Dohrn, 1881 in the Eastern Mediterranean Sea (filled rectangle) and the sampling area (filled triangle) reported in this study

SOLER-MEMBRIVES (2014). The specimen was deposited in the ESFM (Museum of the Faculty of Fisheries, Ege University, İzmir).

\section{RESULTS}

\section{Systematics}

Order PANTOPODA Gerstäcker, 1863

Suborder EUPANTOPODIDA Fry, 1978

Superfamily ASCORHYNCHOIDEA Pocock, 1904

Family AMMOTHEIDAE Dohrn, 1881

Genus TRYGAEUS Dohrn, 1881

Trygaeus communis Dohrn, 1881 (Figs. 2-3)

DOHRN, 1881; BOUVIER,1923; STOCK, 1966:1968; KRAPP-SCHICKEL \& KRAPP, 1975; MUNILLA \& DE HARO, 1984; LEHMAN et al. 2014.

Material examined: 1 $\widehat{~(E F S M-~}$ PYC/2014-1), Mordoğan (İzmir Bay, Turkish

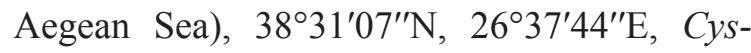
toseira mediterranea Sauvageau, 1912, $0.5 \mathrm{~m}$, 10.08.2014.

Trunk length (frontal margin of cephalic segment to tip of 4th lateral process), $1.34 \mathrm{~mm}$; trunk width (across 1st lateral processes), 0.76 $\mathrm{mm}$.

Remarks: According to DOHRN (1881), BOUVIER (1923) and STOCK (1966) the palps vary in the number of articles from 4 to 7 (LEHMAN $e t$ al., 2014). In this study, the palp has 5 articles (Fig. 3).

Worldwide Distribution: Endemic (Mediterranean Sea) (SOLER-MEMBRIVES \& MUNILLA, 2015).

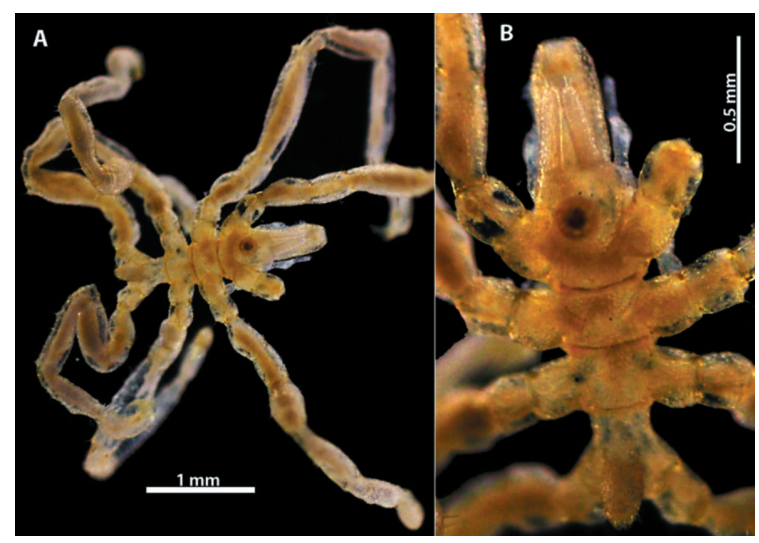

Fig. 2. Trygaeus communis Dohrn, 1881, ठ̂ from Mordoğan, İzir Bay (Aegean Sea, Turkey): A. Dorsal view; B. Trunk, dorsal view

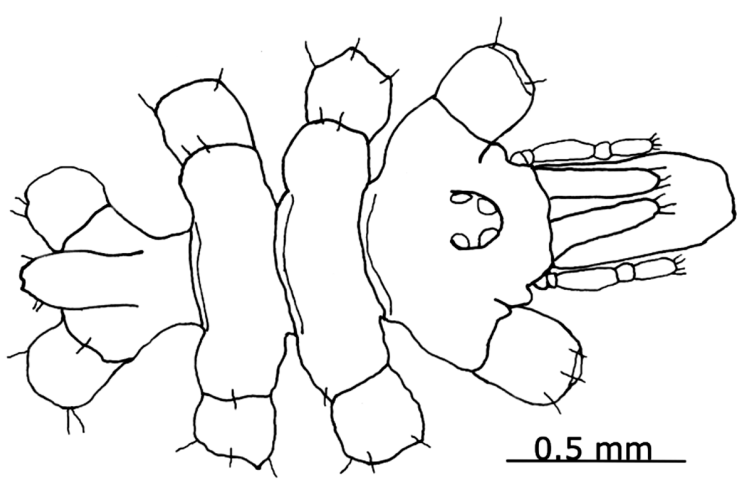

Fig. 3. Trygaeus communis Dohrn, 1881, §̂ from Mordoğan, Izmir Bay (Aegean Sea, Turkey). Trunk, dorsal view 


\section{DISCUSSION}

Most previous records of Trygaeus communis Dohrn, 1881 from the Mediterranean Sea are from the western and central regions: Spanish coast (STOCK, 1966; MUNILLA, 1981:1984:1993:1997; MUNILLA \& DE HARO, 1984; MUNILLA \& NIETO, 1999). French coast (BOUVIER, 1923; BOURDILLON, 1954; ARNAUD, 1987). Italian coast (DOHRN, 1881; FARAGGIANA, 1940; SOYER, 1966; KRAPP, 1973; CHIMENZ et al., 1979; ARNAUD, 1987; LEHMANN et al., 2014). Croatian coast (KRAPP-SCHICKEL \& KRAPP, 1975; ARNAUD, 1987; SCHULLER, 1989; LEHMANN et al., 2014). Tunisian coast (ARNAUD, 1987).

In the Eastern Mediterranean Sea, this species is reported only from Crete Island (Greece) by ARNAUD (1987) (Fig. 1). The new record of $T$. communis reported in this study further extends the known distribution of this species to the Turkish Aegean Sea in the Eastern Mediterranean Sea. With this species, the total number of species is raised to 29 in the Turkish seas, as follows:

Ammothella longipes (Hodge, 1864); A.uniunguiculata (Dohrn, 1881); A. longioculata (Faraggiana, 1940); A. biunguiculata (Dohrn,
1881); A. appendiculata (Dohrn, 1881). Achelia langi (Dohrn, 1881); A. vulgaris (Costa, 1861); A. echinata Hodge, 1864. Tanystylum orbiculare Wilson, 1878; T. conirostre (Dohrn, 1881). Trygaeus communis Dohrn, 1881. Ascorhynchus castelli (Dohrn, 1881). Anoplodactylus petiolatus (Kroyer, 1844); A. pygmaeus (Hodge, 1864); A. virescens (Hodge, 1864); A. digitatus (Böhm, 1879); A. californicus Hall, 1912; A. stocki Bacescu, 1958; A. angulatus (Dohrn, 1881); A. nanus Krapp, Kocak \& Katagan, 2008. Callipallene emaciata (Dohrn, 1881); C. phantoma (Dohrn, 1881); C. spectrum (Dohrn, 1881); C. brevirostris (Johnston, 1836); C. tiberi (Dohrn, 1881). Pycnogonum nodulosum Dohrn, 1881. Endeis charybdaea (Dohrn, 1881); E. spinosa (Montagu, 1808). Nymphon gracile Leach, 1814.

These 29 different species of pycnogonids, out of a total number of 1330 species worldwide (MUNILLA \& SOLER-MEMBRIVES, 2014), represent $2.1 \%$ of all known pycnogonid species. To date, a total number of 56 pycnogonid species are found in the Mediterranean Sea (KOCAK, 2015). Thus, the reported 29 species in the Turkish seas represent $51.7 \%$ of the actual Mediterranean Sea species.

\section{REFERENCES}

ARNAUD, F. 1987. Les pycnogonides (Chelicerata) de Méditerranée: Distribution écologique, batyhmétrique et biogéographie. Mésogée, 47: 37-58.

BOURDILLON, A. 1954. Les pycnogonides de Marseille et ses environs. Rec. Trav. Sta. Mar. Endoume, 12: 145-158.

BOUVIER, E. L. 1923. Faune de France - Pycnogonides. Fédération Française des Sociétés de Sciences Naturelles Publisher. Paris, 70 pp.

CHIMENZ, C., P. M. BRIGNOLI \& G. BASCIANO. 1979. Pantopodi del porto di Civitavecchia e dintorni (Italia centrale). Cah. Biol. Mar., 20: 471-497.

CHIMENZ GUSSO C. \& L. LATTANZI. 2003. Mediterranean Pycnogonida: faunistic, taxonomical and zoogeographical considerations. Bioge- ographia, 24: 251-262.

DOHRN, A. 1881. Die Pantopoden des Golfes von Neapel und der angrenzenden Meeresabschnitte. Von Wilhelm Engelmann Publisher. Leipzig, $252 \mathrm{pp}$.

FARAGgianA, R. 1940. Pantopodi del Mare Ligure. Boll. Mus. Zool. Anat. Comp. Torino, 48: $145-158$.

KOCAK, C. 2015. A checklist of the Pycnogonids (Arthropoda) from Turkish waters. Acta Adriat., 56: 189-198.

KRAPP, F. 1973. Pycnogonida from Pantellaria and Ctania, Sicily. Beaufortia, 21: 55-74.

KRAPP-SCHICKEL, G. \& F. KRAPP. 1975. Quelques traits de l'écologie d'amphipodes et de pycnogonides provenant d'un ilot nord-Adriatique. Vie Milieu, 25:1-32. 
LEHMANN, T., M., HEß \& R.R., MELZER. 2014. Common littoral pycnogonids of the Mediterranean Sea. Zoosyst. Evol., 90: 163-224.

MUNILLA, T. 1981. Contribució al coneixement de la distribució ecològica dels picnogònides a la Costa Brava Catalana. Bull. Ins. Cat. Hist. Nat., 47: 77-86.

MUNILLA, T. 1984. Picnogonidos costeros de Castellón de la Plana. Bol. Asoc. Herpetol. Esp., 8: 13-20.

MUNILLA, T. 1993. Contribuciòn a la picnogonifauna del Mediterraneo Espaňol. Ann. Mus. Civ. Stor. Nat. Giacomo. Doria, 89: 445-455. MUNILLA, T. 1997. Picnógonidos capturados en las campañas Fauna II y Fauna III. Orsis, 12: 65-70.

MUNILLA, T. \& A., DE HARO. 1984. Picnogònids de les Illes Medes. In: J. Ros, I. Olivella, J.M. Gili (Editors). Els sistemes naturals de les Illes Medes. Institut d'Estudis Catalans, Barcelona. pp. 531-536.

MUNILLA, T. \& D. NIETO. 1999. Littoral pycnogo- nids from the Chafarinas Islands (Alboran Sea, western Mediterranean). Vie Milieu, 49: 155-161.

MUNILLA, T. \& A. SOLER-MEMBRIVES. 2014. Pycnogonida. Serie Fauna Ibérica. CSIC Publisher. Madrid, 296 pp.

SCHULLER, S. 1989. Die Pantopodenfauna von Rovinj (Nördliche Adria) und der Jahreszyklus einiger Arten. Bonn. Zool. Beitr., 40: 285-295.

SOLER-MEMBRIVES, A. \& T. MUNILLA. 2015. PYCNOIB: Biodiversity and Biogeography of Iberian Pycnogonids. PLoS ONE, 10: 1-21 SOYER, J. 1966. Sur quelques Pycnogonides du Golfe de Gênes. Doriana, 4: 1-5.

STOCK, J. H. 1966. Sur quelques Pycnogonides de la région de Banyuls ((3e note). Vie Milieu, 17: 407-417.

STOCK, J. H. 1968. Faune marine des PyrénéesOrientales. 6: Pycnogonides. Vie Milieu Suppl., 19: 11-38. 
Novi nalaz roda Trygaeus Dohrn, 1881 i vrste Trygaeus communis Dohrn, 1881 (Arthropoda, Pycnogonida) u Turskoj (istočno Sredozemlje)

\author{
Cengiz KOCAK
}

Kontakt e-pošta: kocakcengiz@gmail.com

\begin{abstract}
SAŽETAK
Tijekom uzorkovanja u turskom dijelu Egejskog mora pronađenisu primjerci vrste Trygaeus communis Dohrn, 1881. U Turskoj su prvi put zabilježeni rod Trygaeus i vrsta Trygaeus communis Dohrn, 1881. godine. Također, rodovi i vrste zabilježeni su po drugi put u istočnom Sredozemlju. $\mathrm{U}$ ovom radu prikazana je karta rasprostranjenosti vrste u istočnom Sredozemnom moru, zajedno s fotografijama i crtežima.

Ovaj zapis dodatno proširuje poznatu zastupljenost ove vrste u Turskoj u istočnom Sredozemlju.

Ključne riječi: Pycnogonida, Trygaeus communis, Turska, istočno Sredozemlje, Sredozemno more
\end{abstract}


